\title{
PSYCHOPATHOLOGY, MEMORY AND QUALITY OF LIFE IN CUSHING'S SYNDROME (EP-17)
}

\author{
A. Santos (1), E. Resmini (1), I. Crespo (1), E. Valassi (1), MA. Martinez (2), P. Pires (3), and SM. Webb (1)
}

(1) Endocrinology/Medicine Department, Hospital Sant Pau, Centro de Investigación Biomédica en Red de Enfermedades Raras (CIBER-ER, Unit 747), IIB-Sant Pau, ISCIII and Autonomous University of Barcelona (UAB). Spain.

(2) University Nursing School (UAB), Hospital Sant Pau. Barcelona. Spain.

(3) INNDACYT, Avda. Europa, 20, planta baja puerta D, 08907 Hospitalet de Llobregat. Spain

\section{INTRODUCTION}

Cushing's syndrome (CS) has been related to higher psychopathology. Most studies agree that hypercortisolism may lead to increased anxiety and depression, which is still present even after biochemical cure. Depression is usually the most prevalent characteristic and has been associated to female sex, older age, higher baseline urinary cortisol, more severe clinical condition and absence of detectable pituitary adenoma.

There is a debate regarding the improvement in depressed mood, anxiety or somatic symptoms after cortisol normalization.

Psychopathology can be related to poor memory performance, and low quality of life.

The aim of this study is to establish the relationship between the three parameters and with subjective symptom perception in CS.

\section{METHODS}

Thirty-six patients in remission of CS and 36 matched controls for age, sex and education years were included in the study. Patients and controls were matched for age, sex and education level. They completed SCL-90R, CushingQoL (only patients), Rey-Osterrieth Complex Figure, and a symptom list ranging from 0 to 10 . They also performed blood tests.

Patients were considered biochemically cured of CS after surgery if adrenal insufficiency was demonstrated or if morning cortisol suppression ( $<50 \mathrm{nmol} / \mathrm{l})$ was observed after $1 \mathrm{mg}$ dexamethasone overnight and if repeated $24 \mathrm{~h}$ urinary free cortisol measures were normal $(<280 \mathrm{nmol} / \mathrm{l})$.

Statistical analysis was performed using student $T$ for parametric variables, Mann Whitney $U$ for non parametric variables, and Pearson's correlations.

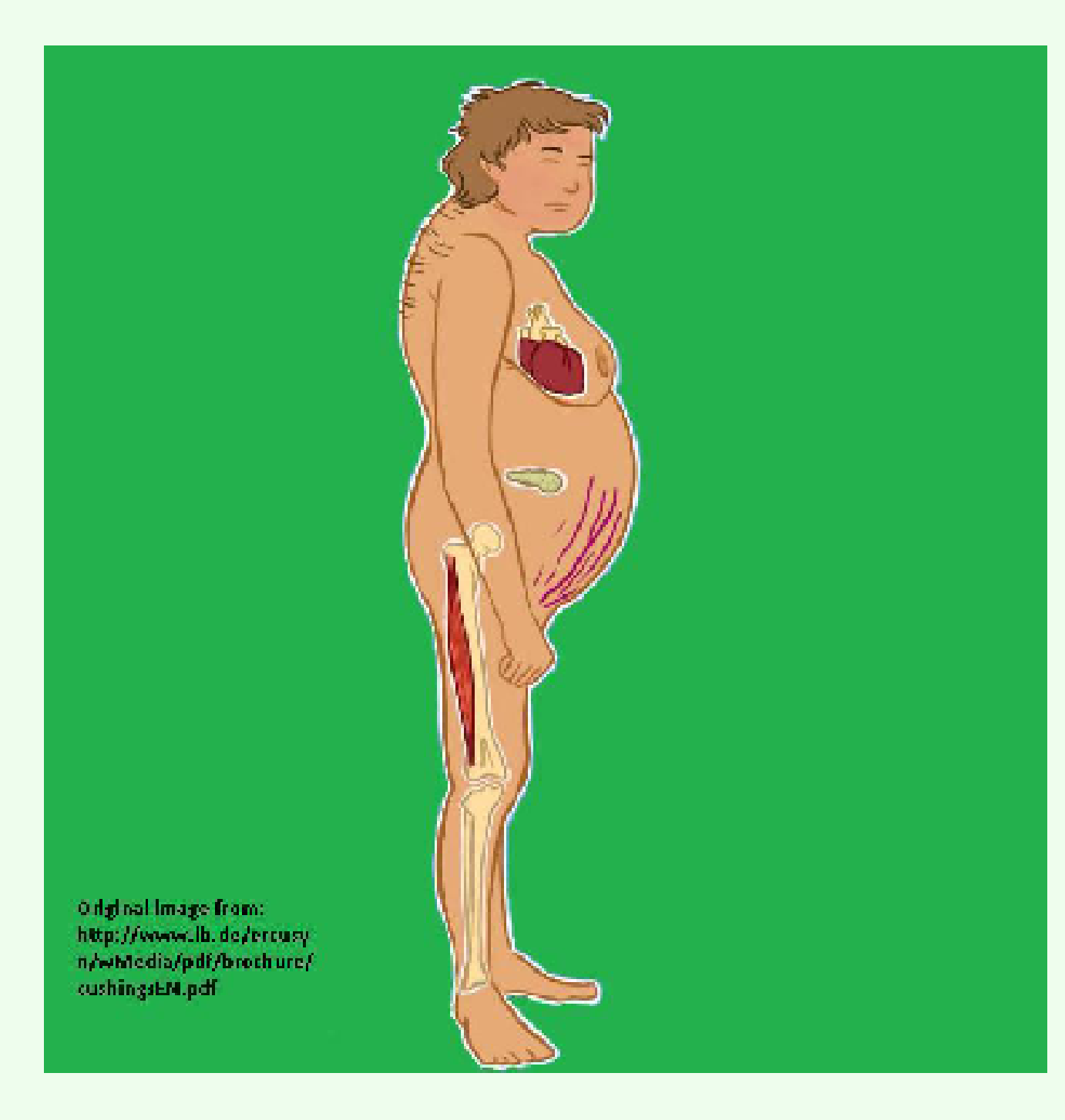

\section{RESULTS}

Patients showed more psychopathology in all the SCL-90R variables (somatization, obsessive-compulsive, interpersonal sensitivity, depression, anxiety, hostility, phobic anxiety, paranoid ideation, psychoticism, global severity index, positive symptom distress index and positive symptom total) in comparison to controls $(p<0.01)$ (Figure 1$)$. They also showed poorer long-term memory performance $(p=0.024)$, compared to controls. Patients also reported a higher severity of symptoms than controls $(p<0.001)($ Figure 2$)$.

Psychopathology did not correlate with memory performance, quality of life or symptoms. The hostility subscale of SCL-90R was positively correlated to blood cortisol levels $(r=0.459, p=0.005)$.

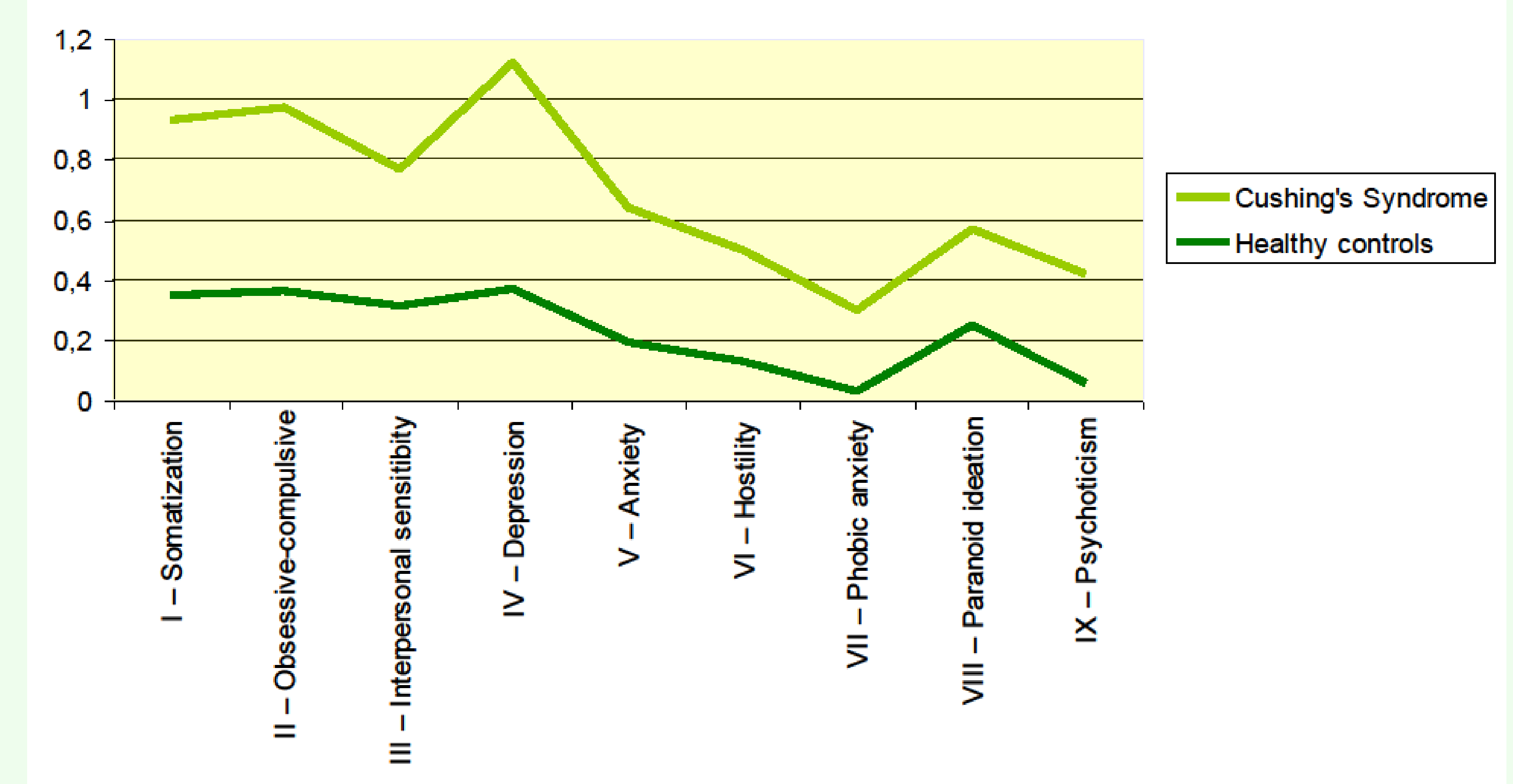

Figure 1. SCL-90R scores in CS patients and healthy controls. Significant differences were found for all scores when comparing patients and controls.



Figure 2. Reported symptoms (rated from 0 to 10) in CS patients and healthy controls. Significant differences were found for all scores except for erectile dysfunction and menstrual irregularity, when comparing patients and controls.

\section{CONCLUSIONS}

CS patients in remission show more psychopathology and memory impairments than healthy controls. These data show that psychological morbidity persists despite normalization of hypercortisolism. Psychopathology was not related to memory performance, quality of life or symptom severity in this sample.
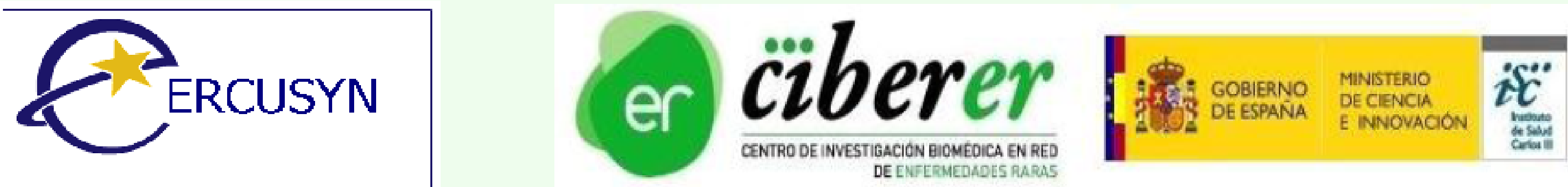